\title{
Gastos hospitalarios derivados de la mala colocación de Catéter Tenckhoff
}

\section{Hospital charges resulting from Tenckhoff Catheter misplacement}

DOI: $10.46932 /$ sfjdv3n1-017

Received in: Dec 30st, 2021

Accepted in: Jan 1th, 2022

\begin{abstract}
Martin Eduardo Avendaño Mejia
Maestro en Administración en Instituciones de Salud (Master in Administration in Health Institutions) Instituto Mexicano del Seguro Social (Mexican Social Security Institute)

Unidad Médica de Atención Ambulatoria- Hospital General de Zona \#1 "Dr. Demetrio Mayoral Pardo" (Ambulatory Care Medical Unit - General Hospital of Zone \# 1 "Dr. Demetrio Mayoral Pardo")

E-mail.: martyned515@gmail.com
\end{abstract}

\begin{abstract}
RESUMEN
En México, la población envejeciendo y los estilos de vida nada saludables, conlleva un incremento en la incidencia de enfermedades crónicas degenerativas, lo cual presenta una serie de nuevos retos en materia de salud, entre los que destaca una demanda creciente de tratamientos de sustitución renal, lo que a su vez conlleva una serie de problemas administrativos. La diálisis peritoneal es la primera terapia de sustitución ofrecida en Oaxaca y para ello se necesita la colocación de un catéter, que cuando esta falla se necesita que se reemplace por otro, para ello el estudio analiza 1,239 pacientes que iniciaban terapia en diálisis peritoneal de las cuales el 24.9\% (309 pacientes) disfuncionaron post colocación, se analizó la posible causa y se analiza los gastos que conllevan la disfunción de ellos.
\end{abstract}

Palabras clave: Costos, Disfunción, Catéter Tenckhoff.

\begin{abstract}
In Mexico, the aging population and unhealthy lifestyles, leads to an increase in the incidence of chronic degenerative diseases, which presents a series of new challenges in health, including a growing demand for substitution treatments. kidney, which in turn leads to a series of administrative problems. peritoneal dialysis is the first substitution therapy offered in Oaxaca and for this the placement of a catheter is needed. When this fails, it needs to be replaced by another, for this the study analyzes 1,239 patients who started therapy in peritoneal dialysis of the which $24.9 \%$ (309 patients) dysfunction post-placement, the possible cause was analyzed and the expenses that lead to their dysfunction are analyzed
\end{abstract}

Keywords: Costs, Dysfunction, Tenckhoff Catheter, Dysfunction, Tenckhoff Catheter.

\section{INTRODUCCIÓN}

La Sociedad Internacional de Nefrología publica a través de sus Guías KDIGO (KIDNEY DISEASE IMPROVING GLOBAL OUTCOMES), define a la Enfermedad Renal Crónica como anomalías de la estructura o función del riñón presentes durante más de 3 meses con implicaciones para la salud. Cuando el usuario llega a la Insuficiencia Renal Crónica terminal es documentado con una tasa de filtrado glomerular $<15 \mathrm{ml} / \mathrm{min}$, donde se requiere empleo de alguna terapia sustitutiva de la función 
renal. Las terapias sustitutivas son el recurso terapéutico de soporte renal en cualquiera de las modalidades: diálisis peritoneal, hemodiálisis o trasplante.

La enfermedad renal crónica (ERC) está relacionada directamente con hipertensión, diabetes y dislipidemias, enfermedades que han alcanzado proporciones epidémicas en nuestro país. La ERC es un proceso multifactorial de carácter progresivo e irreversible que frecuentemente conduce a un estado terminal en el cual la función renal se encuentra lo suficientemente deteriorada como para ocasionar la muerte del paciente o bien para requerir de terapias de sustitución renal. Lo que nosotros vemos es solo una punta de iceberg ya que no contamos una base de datos donde podamos ver las etapas previas de ERC, y por lo que en dichas etapas no manifiesta sintomatología que deriva a una nula visita médica.

\section{DESCRIPCIÓN DEL MÉTODO}

La situación legal y ética del estudio está en virtud del decreto por el que se adicionó el Artículo 4o. Constitucional, publicado en el Diario oficial de la Federación de fecha 3 de febrero de 1983, se consagró como garantía social el Derecho a la Protección de la Salud; reglamentaria del párrafo tercero del Artículo 4o. de la Constitución Política de los Estados Unidos Mexicanos, que a su mención el articulo 23.- En caso de investigaciones con riesgo mínimo, la Comisión de Ética, por razones justificadas, podrá autorizar que el consentimiento informado se obtenga sin formularse escrito, y tratándose de investigaciones sin riesgo podrá dispensar al investigador la obtención del consentimiento informado.

La investigación solo se designará a todo el paciente con colocación de catéter Tenckhoff de los años 2013 al 2018 en el Hospital General de Zona - Unidad Médica De Atención Ambulatoria \#1 "Dr. Demetrio Mayoral Pardo" del Instituto Mexicano del Seguro Social. La muestra se compone 1,239 pacientes que iniciaban terapia en diálisis peritoneal. El instrumento fue documentado por lo que no se necesitó una validación de instrumento.

La cantidad de catéteres que se han colocado año con año van en incremento, en el 2013 se colocaron 158 catéteres y solo ingresaron 103 al programa, en el 2014 se colocaron 177 catéteres e ingresaron al programa 133, en el 2015 ingresaron 187 pacientes e ingresaron al programa 149 para el 2016 colocaron 189 catéteres e ingresaron al programa 157 pacientes, en 2017 se colocaron 225 catéteres ingresaron 180 pacientes al programa, y para el 2018 se colocaron dos 303 catéteres y de los cuales solamente 243 pacientes ingresaron al programa. En la tabla 1 podemos ver la relación que tiene estos datos, y en la tabla 2 una gráfica donde expone los resultados de la colocación de catéteres y los disfuncionales. 
Tabla 1

$\begin{array}{lllll}\text { Años } & \text { Colocados } & \text { Ingreso a Programa } & \text { Disfuncionales } & \% \\ 2013 & 217 & 166 & 51 & 23.5 \\ 2014 & 230 & 187 & 43 & 18.7 \\ 2015 & 261 & 198 & 63 & 24.1 \\ 2016 & 305 & 258 & 47 & 15.4 \\ 2017 & 225 & 180 & 45 & 20.0 \\ 2018 & 303 & 243 & 60 & 19.8\end{array}$

Tabla 2

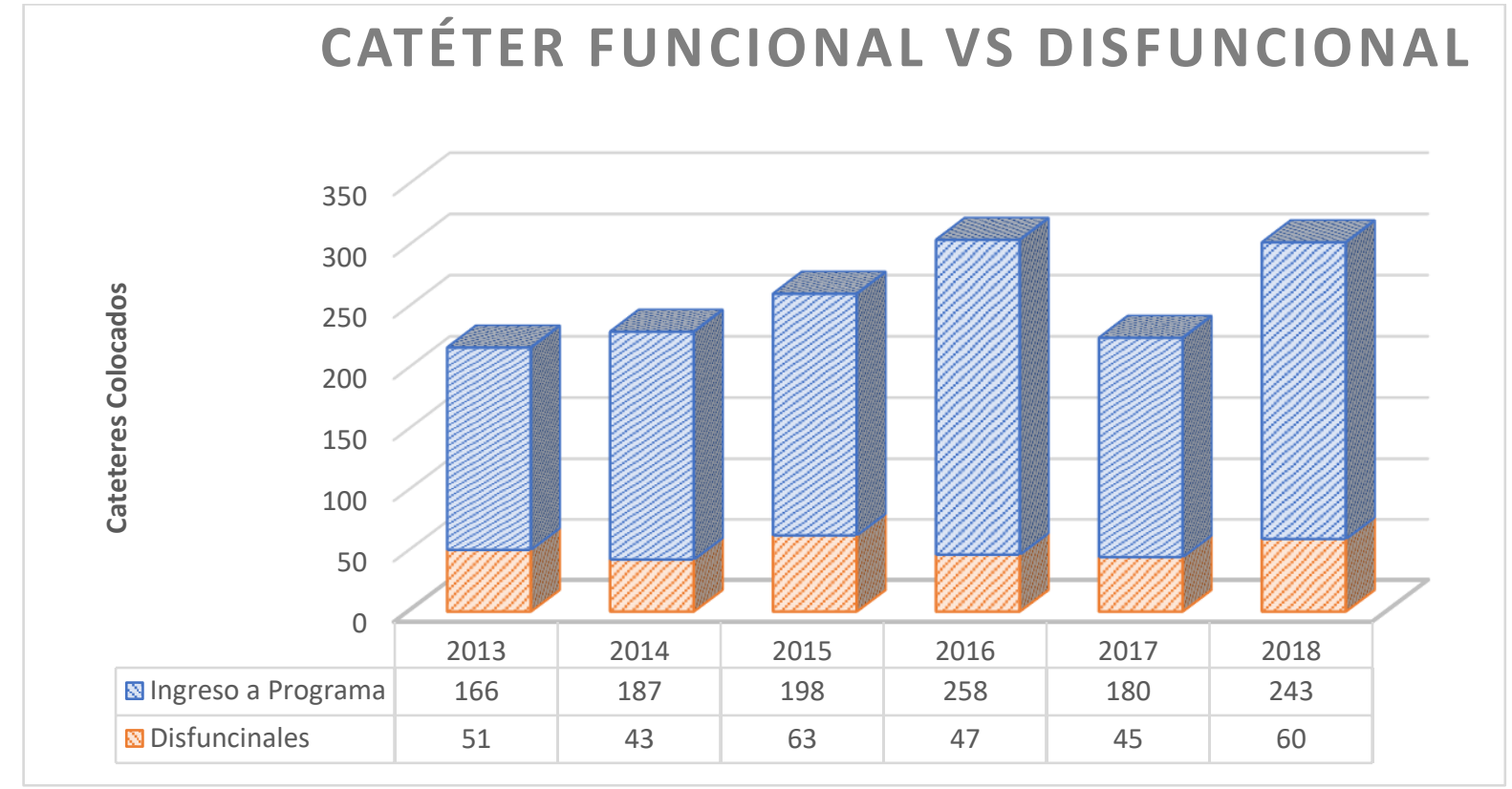

En el cuadro se observa el año consecutivo del 2013 al 2018 cada uno de ellos tiene a su derecha la cantidad de pacientes que disfuncionaron a ello el promedio de día cama en medicina interna es de $\$ 8,333.00$ aquí lo que se hizo fue multiplicar $51 *$ el promedio de día cama por la cantidad de procedimientos quirúrgicos se toma en cuenta que cada intervención quirúrgica de acuerdo a los montos emitidos en el diario oficial \$22,829.00 son por cada intervención quirúrgica a ellos se les multiplicó por el número de pacientes disfuncionales posteriormente ello a la derecha marcamos el promedio de día de estancia cama en medicina interna que es por 3 así que el dato obtenido el día por estancia cama se multiplica por 3 obteniendo el monto de día promedio cama para finalizar se suman tanto el costo de intervención quirúrgica con el costo de día cama en medicina interna y nos da el costo anual obtenido las siguientes cifras. Cabe destacar que los precios de dichos costos de obtiene de los diarios publicados en el Diario Oficial de la Federación, relativo a los costos unitarios de atención en hospitales de segundo nivel. 
tabla 3

\begin{tabular}{|c|c|c|c|c|c|}
\hline \multirow[t]{2}{*}{ Año } & \multirow[t]{2}{*}{$\begin{array}{l}\text { Pacientes con } \\
\text { catéteres } \\
\text { disfuncionales }\end{array}$} & $\begin{array}{lr}\text { Promedio día } \\
\text { cama en } \\
\text { medicina interna }\end{array}$ & \multirow{2}{*}{$\begin{array}{l}\text { Promedio de } \\
\text { recolocaciones por } \\
\text { paciente }\end{array}$} & \multirow{2}{*}{\begin{tabular}{|l} 
Por promedio \\
día cama en \\
medicina interna
\end{tabular}} & \multirow[t]{2}{*}{$\begin{array}{l}\text { Costo anual por } \\
\text { disfunción de catéter }\end{array}$} \\
\hline & & $\$ 8,333.00$ & & & \\
\hline 2013 & 51 & $424,983.00$ & $\$ 1,164,279.00$ & $\$ 1,274,949.00$ & $\$ \quad 2,439,228.00$ \\
\hline 2014 & 43 & $358,319.00$ & $\$ 981,647.00$ & $\$ 1,074,957.00$ & $\$ 2,056,604.00$ \\
\hline 2015 & 63 & $524,979.00$ & $\$ 1,438,227.00$ & $\$ 1,574,937.00$ & $\$ 3,013,164.00$ \\
\hline 2016 & 47 & $391,651.00$ & $\$ 1,072,963.00$ & $\$ 1,174,953.00$ & $\$ \quad 2,247,916.00$ \\
\hline 2017 & 45 & $374,985.00$ & $\$ 1,027,305.00$ & $\$ 1,124,955.00$ & $\$ 2,152,260.00$ \\
\hline 2018 & 60 & $\$ \quad 499,980.00$ & $\$ 1,369,740.00$ & $\$ 1,499,940.00$ & $\$ \quad 2,869,680.00$ \\
\hline
\end{tabular}

Cabe destacar que hay algunos gastos que se agregan por paciente pero que estos son omitidos por lo complejo de algunos casos dejando de solo los gastos ya mencionados en la tabla 3 y reflejados en la gráfica (tabla 4) los gatos que conlleva la disfunción de catéter Tenckhoff.

Tabla 4

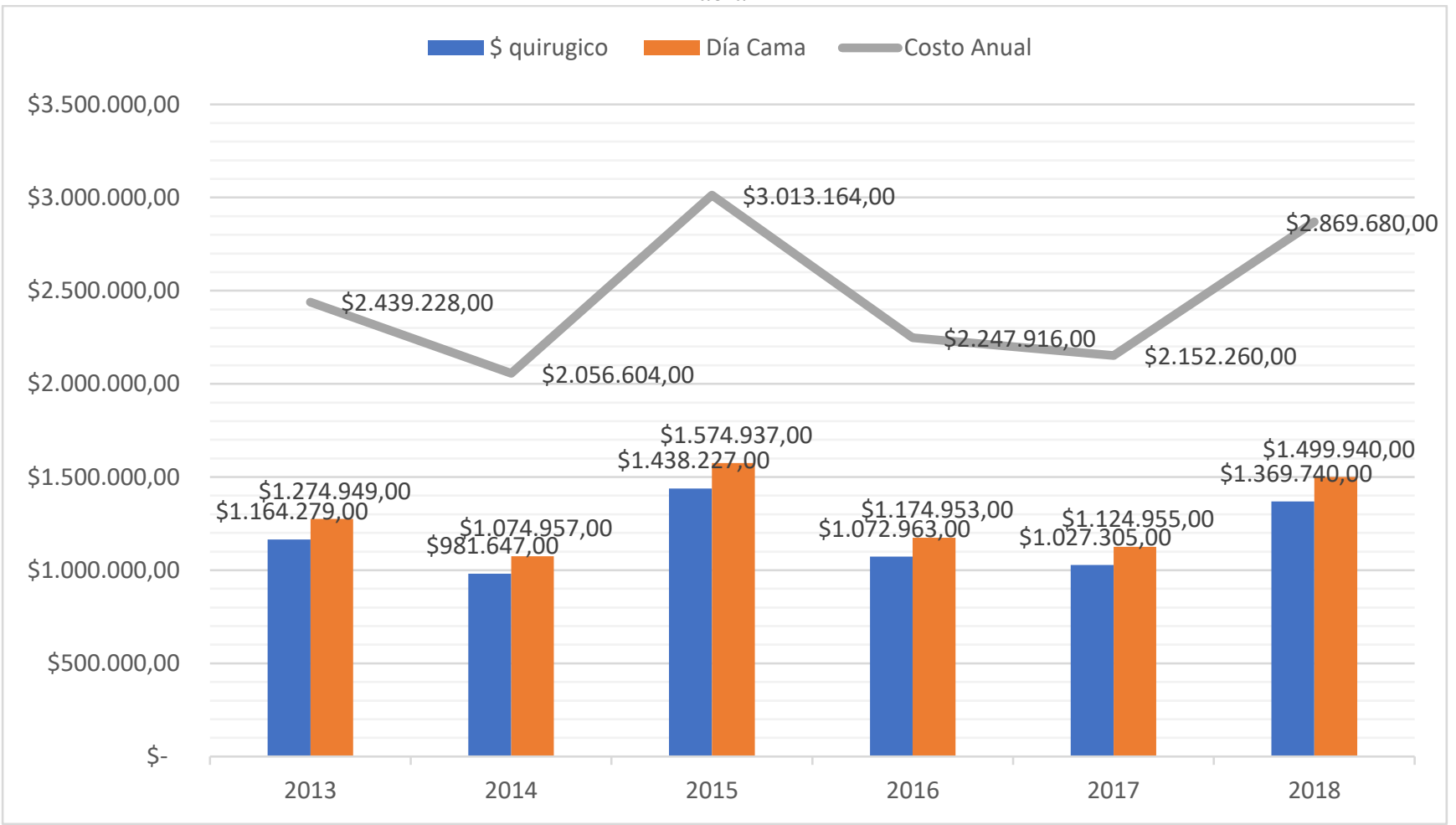

A todo esto, parte del análisis dónde evaluamos el desempeño de cirujanos llegó a la conclusión que lo más conveniente es colocar de manera permanente que solamente los 4 cirujanos con la menor incidencia de disfunciones de catéter sean quienes colocan los catéteres, para poder así disminuir la 
incidencia de catéter Tenckhoff. Datos que podemos interpretar en la tabla 5. Donde se recogen datos de los 1239 usuarios y se conisderan todos los cirujanos (cx) que intervinieron en la colocacion de cateter, y donde el cx 18 fue donde re reunieron a los pacientes que tenian omisiones de papeleria y no se podia corroborar que dicho cirujano fuese el que en realidad lo coloco.

tabla 5

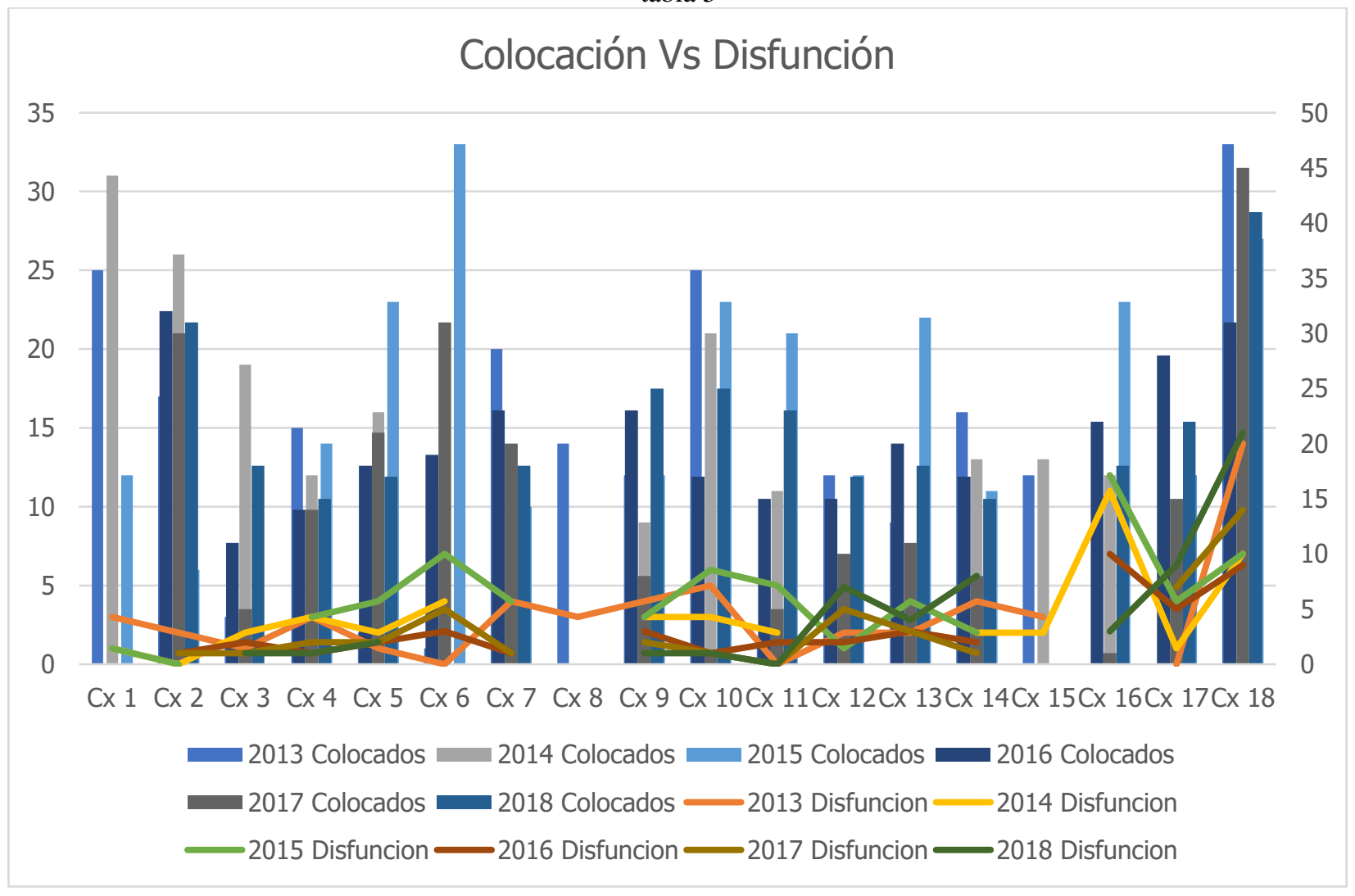

En relación a los costos derivados de la disfunción de catéter Tenckhoff se han enviado directamente al comité de diálisis para su análisis al igual que se ha enviado la propuesta dicha en esta investigación y esperamos una pronta respuesta por parte del comité. 


\section{BIBLIOGRAFÍA}

1.- National Kidney Foundation. K/DOQI clinical practice guidelines for chronic kidney disease: evaluation, classification, and stratification. Am J Kidney Dis. 2002;39(suppl 2):S1-S266.

2.- Diálisis Peritoneal lo que se necesita saber (2007), National Kidney Foundation, http://www.kidney.org/sites/default/files/docs/peritonealdialysis_span.pdf

3.- Kidney Disease: Improving Global Outcomes (KDIGO) CKD Work Group. KDIGO 2012 clinical practice guideline for the evaluation and management of chronic kidney disease. Kidney Int Suppl. 2013;3:1-150.

4.- $\quad$ Levey AS. A decade after the KDOQI CKD guidelines. Am J Kidney Dis. 2012;60:683-685.

5.- $\quad$ Kasiske BL, Eckardt KU. Evidence and outcomes in CKD. Am J Kidney Dis. 2012;59:492-494.

6.- $\quad$ Liu WJ. Complications after tenckhoff catheter insertion: a single-centre experience using multiple operators over four years. Perit Dial Int 2010.

7.- $\quad$ L. Hernando Avendaño, Nefrología Clínica, tercera edición, editorial panamericana, año 2009, 799-810.

8.- Jesús Montenegro, Ricardo Correa-Potter, Miguel C. Riela. Tratado de Diálisis Peritoneal. Primera edición, año 2009, 655 páginas.

9.- Dres. J. Campos Sotas, R. Gianni Davis, P. Leal, J.C. Olivares de la Fuente, J. Rodríguez, V. Castro Giren Herrera. Aspectos quirúrgicos en el uso de catéter Tenckhoff Unidad de Urología, Servicio de Cirugía, Hospital de Niños.

10.- $\quad$ http://diariooficial.gob.mx/nota_detalle.php?codigo $=5554895 \&$ fecha $=22 / 03 / 2019$ Revisado el 24/08/19 